Marquette University

e-Publications@Marquette

School of Dentistry Faculty Research and

Publications

Dentistry, School of

4-1-2011

\title{
Moving an incisor across the midline: A treatment alternative in an adolescent patient
}

Jose A. Bosio

Marquette University, jose.bosio@marquette.edu

T. Gerard Bradley

Marquette University, thomas.bradley@marquette.edu

Arthur F. Hefti

Marquette, arthur.hefti@marquette.edu

Accepted version. American Journal of Orthodontics and Dentofacial Orthopedics, Vol. 139,No. 4, (April, 2011): 533-543 . Publisher Link. (C) 2011 American Association of Orthodontists. Published by Elsevier Inc. Used with permission. 
Marquette University

\section{e-Publications@Marquette}

\section{Dentistry Faculty Research and Publications/School of Dentistry}

This paper is NOT THE PUBLISHED VERSION; but the author's final, peer-reviewed manuscript.

The published version may be accessed by following the link in the citation below.

American Journal of Orthodontics and Dentofacial Orthopedics. Vol. 139. No. 4. (April, 2011): 533-543. DOI. This article is (C) Elseiver and permission has been granted for this version to appear in e-Publications@Marquette. Elsevier does not grant permission for this article to be further copied/distributed or hosted elsewhere without the express permission from Elsevier.

\section{Contents}

Abstract... 2

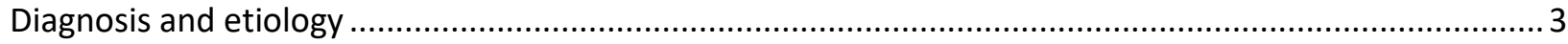

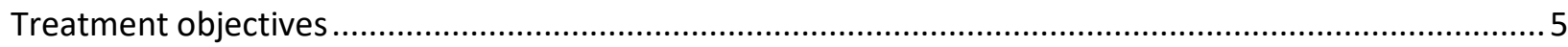

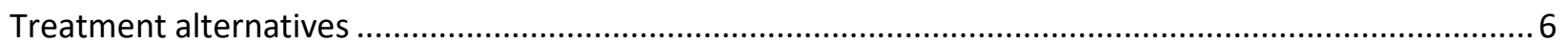

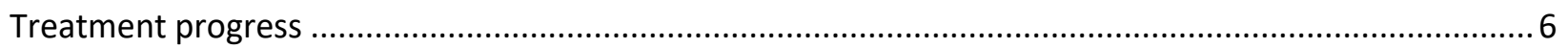

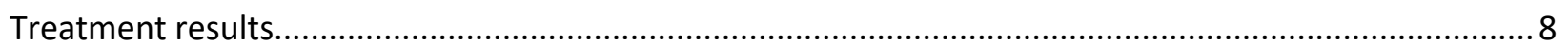

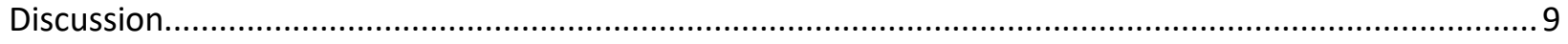

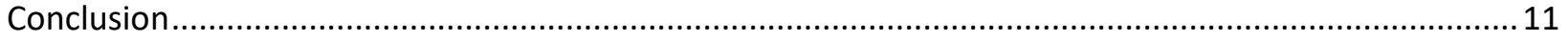

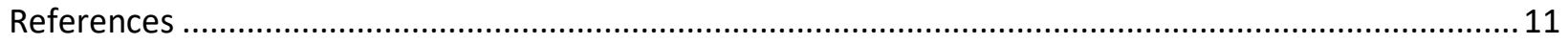




\title{
Moving an incisor across the midline: $A$ treatment alternative in an adolescent patient
}

\author{
Jose A. Bosio
}

Department of Biological Sciences, Marquette University School of Dentistry, Milwaukee, WI

T. Gerard Bradley

Program Director of Orthodontics, Department of Developmental Sciences, Marquette University School of Dentistry, Milwaukee, WI

Arthur F. Hefti

Surgical Sciences, Marquette University School of Dentistry, Milwaukee, WI

\begin{abstract}
A 13-year-old sought treatment for a severely compromised maxillary left central incisor and an impacted fully developed left canine. Extraction of both teeth became necessary. As the key component of the revised comprehensive treatment plan, the right maxillary central incisor was moved into the position of the left central incisor. All other maxillary teeth were moved mesially to close any gaps. Active orthodontic treatment was completed after 34 months. Frenectomy, minor periodontal surgeries, and bonded lingual retainers were used to improve aesthetics and stabilize the tooth positions. The patient was pleased with the treatment outcome. Cone-beam computed tomography provided evidence that the tooth movement was accompanied by a deviation of the most anterior portion of the median palatine suture. This observation may make relapse more likely if long-term retention cannot be ensured. Root resorption was not observed as a consequence of the major tooth movement.
\end{abstract}

Moving an incisor to the contralateral side is a rarely used orthodontic procedure. Only a few cases have been reported in the literature. ${ }^{1}, 2,3,4,5$ Two reports suggested a higher relapse tendency than for other types of tooth movement, but the long-term outcome of the procedure remains to be assessed in controlled clinical studies. ${ }^{1}$ - All published cases with midline crossing were initiated during the mixed dentition and treatment was completed at approximately 12 years of age. $1,2,3,4,5$

Follin et al used Beagle dogs to investigate the movement of incisors as a function of animal age and median palatal suture (MPS) closure. ${ }^{-}$In their study, the movement of the tooth appeared to be faster in old animals, but there was a much higher degree of root resorption. In addition, histology suggested differences among the tooth movements that were related to the status of MPS closure. In young animals the suture was dislocated in front of the test tooth, whereas in old animals with closed MPS the tooth moved across the MPS. -

Thus, the feasibility of relocating a maxillary central incisor across the midline has been demonstrated previously. $1,2,3,4, \underline{5}$ However, to the best of our knowledge, no case has been documented showing this 
type of tooth movement in the permanent dentition of an adolescent patient. The present case report demonstrates the feasibility of closing a wide gap in the aesthetic zone by bilateral shifting of several teeth mesially, in particular a central incisor that was moved across the midline. At treatment completion, cone-beam computed tomography (CBCT) was used to show the relative position of the relocated incisor, the incisive foramen, the nasopalatine canal, and the MPS. In addition, the presence or absence of root resorption was investigated.

\section{Diagnosis and etiology}

A 13-year-old Brazilian boy visited the orthodontic clinic because he had "one missing tooth, and the other teeth were too far forward." He suffered a bicycle accident when he was 8 years old, receiving minor facial bruises. The accident traumatized the maxillary left central incisor, requiring partial pulpotomy. He had a thumb-sucking habit until age 2 years. He did not report any other dental or medical problems, nor was he taking any medication. One week prior to the orthodontic consultation, he saw a general dentist and no caries were diagnosed. At the initial examination and throughout treatment, he maintained good oral hygiene.

At the first orthodontic examination, the patient had a convex soft tissue profile with a retrognathic mandible ( Fig 1, Fig 2, Fig 3, Fig 4). The nasolabial angle was normal to obtuse, and the lower lip was retrusive to Rickett's E plane. From the frontal view, his face was slightly asymmetrical, with the right side being a little longer than the left side. When smiling, the patient presented uneven gingival margin levels. The incisal edges also were not level. The crown of the injured left central incisor was shorter than the right one. Intraoral and dental cast examinations showed a molar full-cusp angle Class II on the right and left. The right canine was also in a Class II relationship. The left maxillary canine had not yet erupted. An overjet of $11 \mathrm{~mm}$ was measured and, as a result of the overjet, the anterior teeth were not in contact. The patient's tongue posture habit likely contributed to this situation. The left maxillary lateral incisor was in palatoversion, possibly due to the unerupted left maxillary canine. Both maxillary first molars were rotated mesially. Mild spacing was observed between the premolars in the mandibular arch. Periodontal and oral mucosal tissues appeared healthy.

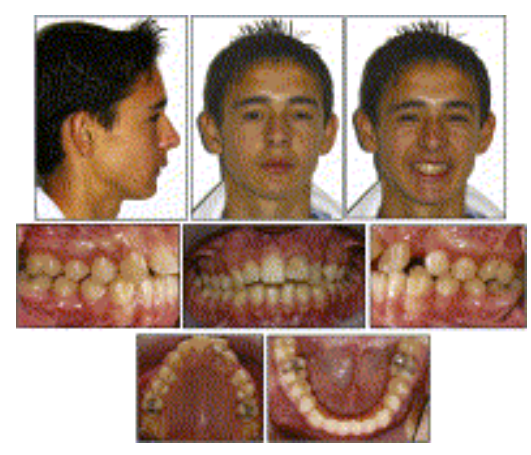

Fig 1 Pretreatment photographs. 


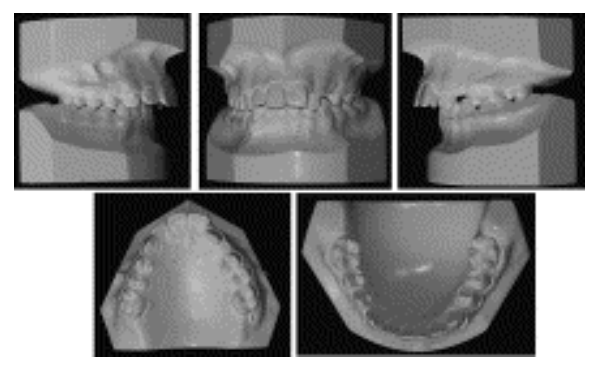

Fig 2 Pretreatment dental casts.

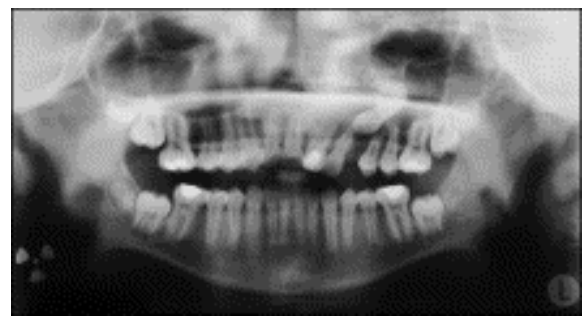

Fig 3 Panoramic radiograph.

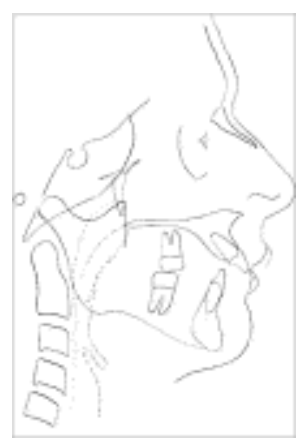

Fig 4 Pretreatment cephalometric tracing.

The panoramic radiograph ( $\underline{\mathrm{Fig} 3}$ ) suggested the presence of a maxillary sinus retention cyst in the area of the maxillary left incisors and canine. An endodontist diagnosed internal resorption of the maxillary left central incisor. The fully developed left maxillary canine was impacted. However, it appeared to push the left lateral incisor root buccally, promoting palatoversion of the lateral incisor crown. Development and eruption of all second molars were incomplete. All third molars were developing and not erupted. The pretreatment cephalometric tracing measurements showed an ANB angle of $5^{\circ}$ (Fig 4 Table 1). A horizontal growth pattern was present, even though the antegonial notch was very pronounced, and the anterior portion of the mandible had a vertical tendency. Maxillary incisors were extremely proclined (U1-FH, $\left.129^{\circ}\right)$, but the mandibular incisors were within normal limits (L1-MP, $\left.91^{\circ}\right)$.

Table 1Initial, pretreatment and post-treatment cephalometric measurements.

\begin{tabular}{|c|l|l|l|}
\hline Analysis & $\begin{array}{l}\text { Initial } \\
\text { Jul 7, 2003 }\end{array}$ & $\begin{array}{l}\text { Pretreatment } \\
\text { Oct 18, 2004 }\end{array}$ & $\begin{array}{l}\text { Posttreament } \\
\text { Aug 09, 2007 }\end{array}$ \\
\hline Skeletal & & & \\
\hline Ba-S-Na & $145^{\circ}$ & $145^{\circ}$ & $144^{\circ}$ \\
\hline SNA & $74^{\circ}$ & $73^{\circ}$ & $71^{\circ}$ \\
\hline
\end{tabular}




\begin{tabular}{|l|l|l|l|}
\hline SNB & $68^{\circ}$ & $68^{\circ}$ & $67^{\circ}$ \\
\hline ANB & $6^{\circ}$ & $5^{\circ}$ & $4^{\circ}$ \\
\hline FH-NPg & $86^{\circ}$ & $85^{\circ}$ & $84^{\circ}$ \\
\hline FH-MPI & $24^{\circ}$ & $23^{\circ}$ & $26^{\circ}$ \\
\hline Y-Axis (FH-SGn) & $58^{\circ}$ & $58^{\circ}$ & $60^{\circ}$ \\
\hline Facial Axis (BaN-CCGn) & $87^{\circ}$ & $88^{\circ}$ & $86^{\circ}$ \\
\hline Wits & $3 \mathrm{~mm}$ & $7 \mathrm{~mm}$ & $1 \mathrm{~mm}$ \\
\hline Dentition & & & \\
\hline U1-FH & $125,5^{\circ}$ & $129^{\circ}$ & $107^{\circ}$ \\
\hline U1-NA & $35^{\circ}$ & $40^{\circ}$ & $22^{\circ}$ \\
\hline U1-L1 & $122^{\circ}$ & $117^{\circ}$ & $126^{\circ}$ \\
\hline L1-MPI & $89^{\circ}$ & $91^{\circ}$ & $101^{\circ}$ \\
\hline L1-NB & $18^{\circ}$ & $18,5^{\circ}$ & $29^{\circ}$ \\
\hline Soft tissue & & & \\
\hline Upper lip to S-line & $1 \mathrm{~mm}$ & $1 \mathrm{~mm}$ & $-2 \mathrm{~mm}$ \\
\hline Upper lip to E-line & $-2 \mathrm{~mm}$ & $-2 \mathrm{~mm}$ & $-6 \mathrm{~mm}$ \\
\hline Lower lip to E-line & $-4 \mathrm{~mm}$ & $-4 \mathrm{~mm}$ & $-3 \mathrm{~mm}$ \\
\hline & & & \\
\hline
\end{tabular}

\section{Treatment objectives}

The primary treatment objective was to establish a harmonious facial profile and a physiologic occlusion. Initially, the tongue posture habit would be treated using tongue spurs soldered to a mandibular lingual arch. This would be followed by the extraction of the maxillary right first premolar to reduce the overjet and to finish the case in bilateral molar Class II and canine Class I relationships on the right side. Uncovering the maxillary left canine would allow for integration and alignment of that tooth in the dental arch.. Since the maxillary left central incisor was severely compromised, no effort would be made to save it. The treatment plan also included moving the maxillary left lateral incisor into the position of the left central incisor and the maxillary left canine into the position of the lateral incisor. Both left lateral incisor and canine would be cosmetically restored to match as closely as possible, the shape, size, and color of the right central and lateral incisors. The maxillary left first premolar would be restored to function as a canine.

The mandibular dentition would be aligned ideally, and the spaces closed. The overjet would be reduced and a normal overbite would be established after eliminating the tongue posture habit. The benefits to the patient of this treatment plan would include a natural occlusion, with no implants or crown or bridge work needed in the area of the left central incisor. The disadvantages of the treatment plan would reveal themselves toward the final treatment stages. In fact, mismatching tooth shape, color, and size are often contributing factors for failing canine-substitution cases. $\underline{12}$ Poorly controlled retention could also lead to an opening of the extraction space.

It was recommended that the oral surgeon assess the suspected maxillary sinus retention cyst at the time of the canine exposure, and if necessary remove it (it is usually not necessary).. Extraction of the third molars should be re-evaluated at the final assessment of orthodontic treatment. 


\section{Treatment alternatives}

Extraction of both maxillary first premolars was considered for overjet reduction. It would allow retraction of the maxillary canines (after the left canine exposure and alignment) into a Class I relationship. Opening the space for an implant at the end of the treatment in the area of the maxillary left central incisor would be included in this option.

Bilateral first premolar extractions were also considered, along with transplantation of a third molar to the area of the incisor. 10 This option was not considered further because of the high risk of failure associated with tooth transplants, lack of pertinent professional expertise, and excessive costs.

With the extraction of the maxillary left central incisor and the mesial movement of the left lateral incisor into the position of the central incisor, it was expected that the canine would erupt on its own without the need for surgical exposure. However, when the tooth follicle is located on the palatal aspect of the dental arch, spontaneous canine eruption is less likely to occur. $\frac{11}{}$ Finally, the patient's young age was also considered a disadvantage because of the long waiting period until implant placement (growth completed) and/or subsequent restorative work could take place.

\section{Treatment progress}

Since financial considerations were an important issue, the patient was referred to Pontifical Catholic University Dental School in Curitiba, Brazil. Surgical canine exposure was scheduled 5 months after initial consultation. The surgeon determined at the time of exposure that the canine could not be saved because of the cyst's massive size, the tooth's location, and the proximity of the lateral incisor (Fig 3 ). Thus, the canine was removed along with the cyst. When the patient returned to the orthodontic clinic 11 months later, new records were taken ( $\mathrm{Fig} 5$, Fig 6 ). The full eruption of the second molars and the palatal position of the maxillary left lateral incisor were noted. The changes required the development of a new treatment plan. The decision was to move the right central incisor across the maxillary midline to the position of the left central incisor. As a consequence, on the maxillary right side the lateral incisor would be moved to the position of the central incisor, the canine would be moved to the position of the lateral incisor, and the first premolar would be substituted for the canine. On the left side of the maxilla, the first premolar would replace the canine, and the lateral incisor would remain in its natural position. All permanent maxillary anterior teeth would have to be restored at the end of the treatment. The revised treatment plan was approved by the patient and his parents and orthodontic treatment was initiated.

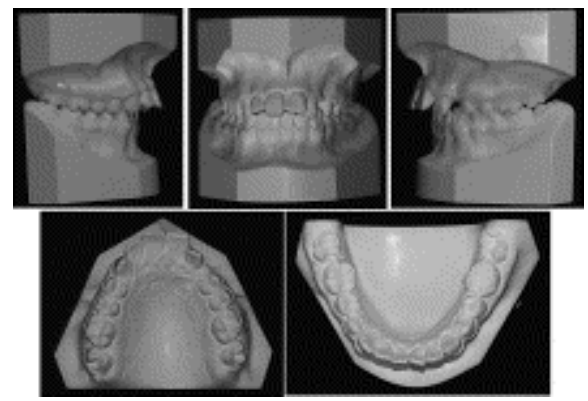

Fig 5 Progress dental casts. 


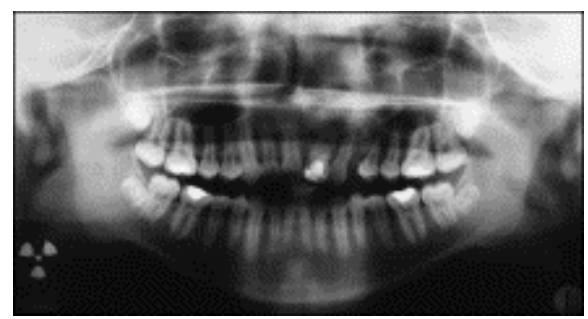

Fig 6 Progress radiograph.

A maxillary left central incisor bracket was bonded on the right central incisor to control the inclination of the root. Roth prescription brackets (0.018-in slot) were placed first on the maxillary teeth. Bands for the construction of the mandibular lingual arch with spurs were placed, and the appliance was inserted on the following visit. Two months later, the mandibular brackets were bonded. After leveling and aligning, a standard stainless steel arch wire $(0.016 \times 0.022$-in) was inserted; width reduction of the maxillary left central incisor crown was initiated and continued until crown removal was indicated. The crown was kept for aesthetic purposes while the space was being closed.

As the treatment progressed, the maxillary left central incisor space was closed with an elastomeric chain. To maintain anchorage on the left side, Class II interdental elastics were used. Mandibular lingual arch bands with spurs were removed 5 months after insertion because of patient complaints. Consequently, an open bite developed in the anterior region because of the failed correction of the tongue posture habit and the simultaneous extrusion of posterior teeth ( $\underline{\text { Fig } 7, A) . ~ T o ~ h e l p ~ c o n t r o l ~ t h e ~}$ tongue habit, bondable minispurs were inserted on the palatal surface of the maxillary lateral and central incisors. Six months before finishing treatment, space was maintained to shape the right lateral incisor into a right central incisor ( $\mathrm{Fig} 7, \mathrm{~B})$. Also, intrusion bends were placed in the wires to correct the open bite. Periapical radiographs confirmed that bone was present mesial to the left maxillary lateral incisor (Fig 7, C). Again, because of the lack of financial resources, a temporary composite restoration was performed to improve aesthetics and facilitate finishing (Fig 7, D).

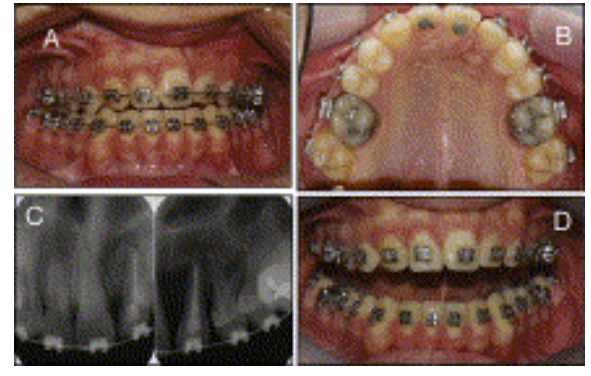

Fig 7 A, Mandibular lingual arch bands with spurs were removed 5 months after placement because of patient complaints, and open bite developed in anterior region B, Bondable minispurs were placed on maxillary lateral and central incisors to control tongue habit, and intrusion bends placed in wires to correct the open bite. Six months before finishing treatment, space was maintained to shape right lateral incisor into right central incisor. C, Periapical radiographs confirmed that bone was present mesial to left maxillary lateral incisor. D, A temporary composite restoration was performed to improve aesthetics and facilitate finishing.

Individualized intrusion of all maxillary premolars was performed to obtain an optimal level for the marginal gingival contours. In addition, increasing the width and length of the crowns using resin 
veneers led to optimal aesthetics. $\underline{\underline{12}}$ The mandibular arch had the minor crowding resolved with routine arch wires and a small amount of interdental enamel reduction of the anterior teeth.

Recommendations for additional improvement in aesthetics and a periodontal consultation were made. The patient was very receptive and cooperative. The treatment was completed after 34 months.

\section{Treatment results}

Given the unusual circumstances and the unavoidable treatment plan changes, the overall result was excellent. Improvement of the patient's facial harmony and profile were achieved. The left maxillary central incisor and canine required extraction, and the resulting spaces were closed. Molar class II occlusion was maintained bilaterally. A better interdigitation of the posterior segment was not achieved because of the distal rotation of the maxillary first molars. Ideal anterior overjet and overbite were attained, which facilitated restoration with composite resin build-ups. Bonding a left central incisor bracket on the right central incisor tooth proved to be a good choice because of the bodily movement of the tooth, without inclination of the crown. Only minimal vertical space was lost in the process of periodontal surgical healing and anterior tooth restoration. These spaces were needed for composite build-ups in the maxillary premolar area. Excessive gingival display was present at the time of appliance removal ( $\mathrm{Fig} 8, \underline{\mathrm{Fig}}$ ). In addition, the labial frenum insertion had moved along with MPS and incisive papillae ahead of the central incisor and now deviated to the left side. Gingival recontouring was performed to create an aesthetically more acceptable result. $. \underline{13}, \underline{14}$ At the same appointment, a frenectomy was performed to prevent frenal pull and future gingival recession. Tooth alignment, contact points, and occlusion were adjusted, according to the teeth present, as close as possible to the ideal situation. A pleasing soft tissue balance resulted (Fig 10).

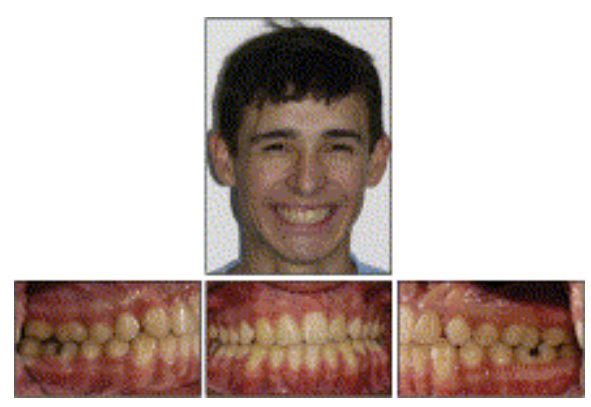

Fig 8 At appliance removal, excessive gingival display was noted. Labial frenum insertion had moved along with MPS and incisive papillae ahead of central incisor and deviated to left side.

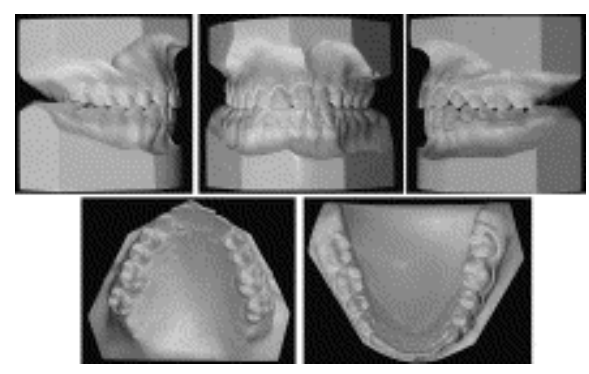

Fig 9 Final dental casts. 


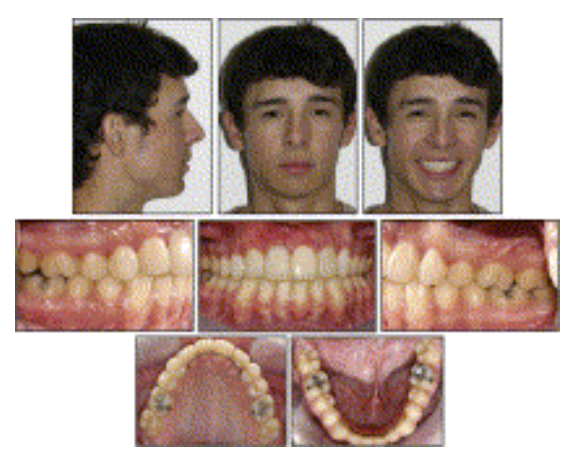

Fig 10 Final photographs.

CBCT and the macroscopically visible, deviated incisive papilla suggested a shift of the MPS toward the affected side (Fig 11). Furthermore, root resorption was minimal and the roots of all anterior teeth were well angulated and aligned. The ANB angle was reduced after treatment because the A-point moved significantly distally as a result of the palatal movement of the incisors (ig 12)

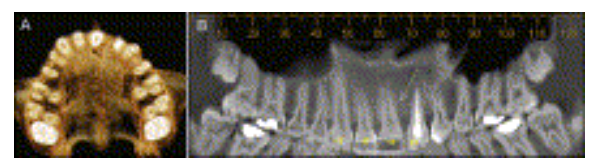

Fig 11 CBCT suggested a shift of the MPS toward the affected side. Root resorption was minimal and the roots of all anterior teeth were well angulated and aligned.

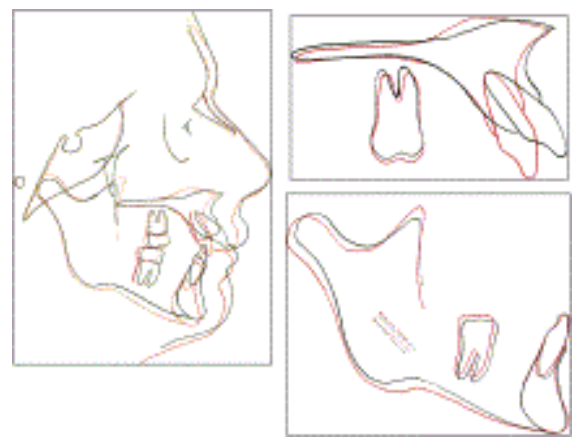

Fig 12 Superimposed tracings (initial in black, final in red).

The retention protocol is as important as the treatment itself. A stainless steel coaxial 0.021-in wire was bonded on the lingual side of the maxillary anterior teeth to prevent spaces from opening. The wire was bonded on the day of appliance removal and was removed only to perform the anterior composite build-ups. A circumferential Hawley retainer with a hole in the acrylic to help the patient direct the tongue while swallowing was also used in the maxillary arch. Mandibular retention was achieved with a canine-to-canine bonded 0.036 -in stainless steel fixed retainer.

\section{Discussion}

A small number of case reports have shown that incisor movement to the contralateral side can be employed under careful consideration of its biological limits. These cases were in young patients, who were in the early mixed dentition stage at the start of treatment and had open palatal sutures. No cases have been reported in which this type of tooth movement was attempted in the permanent dentition. The present case was performed in an adolescent patient and confirmed the outcome of previous 
reports. In particular, it corroborated the inability to move teeth across the suture. $\frac{1}{2}, \underline{3}, \underline{4}, \underline{5}, \underline{6}$ The failure to cross the suture resulted in substantial deviations of the incisive papilla and labial frenum -that is, a rotation around the incisive foramen of the anterior portion of the midpalatal suture occurred. The incisive foramen is the palatal orifice of the nasopalatine canal. Typically, this anatomical landmark is situated at the midpalatal suture, slightly posterior to the central incisors. $\frac{15}{15}$ The nasopalatine canal is the lead structure for the nasopalatine nerve and the end branches of the nasopalatine artery. Moving a tooth to the contralateral side of the maxilla could affect the course of the canal and its content, leading to adverse clinical symptoms. CBCT imaging (Fig 11) suggested that the tooth movement had little or no effect on the position of the incisive foramen. In addition, axial views on CBCT showed that the spatial course of the nasopalatine canal was relatively unchanged. Therefore, it appeared that the orthodontic correction included the teeth, periodontal tissues, and related attached buccal mucosa but did not affect the processes of the hard palate.

Closing the large gap that resulted from losing 2 teeth in a single quadrant required bilateral movement of teeth mesially. As a result, the position of the maxillary left first premolar remained a concern. Moving it to its final position was associated with fenestration of the alveolar bone and partial exposure of the buccal root. Dehiscence and fenestrations of the alveolar bone occur naturally quite frequently. $\frac{16}{}$ However, in the presence of an inadequate tooth brushing technique, they can contribute to the development of periodontal recession with well-known consequences, including sensitivity, caries, and ultimately periodontal surgery. $\frac{17}{}$

Unlike with implants, there are no risks of increasing the degree of infraocclusion during and after completion of growth or of significant marginal bone loss at tooth surfaces adjacent to the implant as described by Thilander et al. $\underline{\underline{18}}$

When a lateral incisor is substituted for a central incisor, the canine takes over the position of the lateral incisor. In this situation, the orthodontist must disregard the incisal edges of these teeth as a guide for final tooth positioning and focus on the location of the gingival margin. The mutual positions of the 6 maxillary anterior teeth, their relative size, and their position relative to the upper lip play important roles in the aesthetic appearance of the patient. ${ }^{14}$ In addition, display and level of the gingiva must be considered. $\underline{19}$ Creation of an aesthetic gingival appearance was difficult in the case presented here. We used a multistep procedure to achieve an acceptable result, including intrusion of the right first premolar, extrusion of the right canine, and intrusion of the lateral incisor. $\underline{13}$ Further improvement was obtained by gingival recontouring.

Using the crown of the extracted central incisor to maintain aesthetics proved to be an ideal choice. The progressive reduction of the mesiodistal dimension was performed as necessary. However, the case planning would have been better had a diagnostic wax-up been used. $\underline{\underline{14}}$ It would have permitted an even distribution of the spaces before the treatment.

The choices for restoring the anterior teeth could include composite bonding, porcelain veneers, bonded all-ceramic crowns, luted all-ceramic crowns, or metal ceramic crowns. $\frac{14}{}$ Generally, the treatment of choice is the most conservative restoration that satisfies the patient's aesthetic requirements. $\underline{20}$ Thus, the materials used for the restoration in this case were composite resin build-ups due to their availability and the ability to create ideal tooth morphology, even considering the need for frequent repair. $\frac{12}{}$ In addition, the age of the patient would also contraindicate the use of veneers and crowns. 
No signs of temporomandibular joint problems were present at the beginning of treatment, and, as expected, there were no symptoms throughout the course of treatment and into retention. $\stackrel{21}{ }$ Contrary to Follin's study, $\underline{\underline{2}}$ we recommend the bonded retainer to remain indefinitely, even after suture closure, in order to maintain the achieved result. The bilateral cusp-fossa relationship of the first and second molars bilaterally and left first premolar areas ( $\underline{\text { Fig } 9}$, Fig 10) were not ideal. The large mesiodistal size of the anterior remaining teeth was the likely reason for the malocclusion, which did not compromise the functional outcome. Last but not least, patient self-esteem was greatly improved as a result of the substantial improvement of aesthetics.

\section{Conclusion}

This case report described the closure of a 2-tooth space without using implants or bridge placement in an adolescent patient. The core of the treatment plan included the movement of a right central incisor to the left central incisor location. An initial treatment plan could not be completed because an impacted canine, which was planned for arch integration, had to be extracted. An alternative treatment plan, combining tooth movements from the right to the left side of the maxilla and mesialization of the remaining teeth on the left side, was performed. No significant root resorption was observed as a consequence of the major tooth movement. The anterior portion of the midpalatal suture, the incisive papilla, and the maxillary labial frenum also moved with the relocated tooth. Soft tissue discrepancies were addressed using gingivectomy, gingival recontouring, and frenectomy. Bonded lingual retainers were used to further improve aesthetics and stabilize the resulting tooth positions. Retention must be regularly checked because the space between right central (now left central) and left lateral incisors could re-open.

The periodontal and restorative procedures were performed in a charitable fashion immediately after appliance removal by Drs. Silvia R.B. Pontes and Carmen Storrer of Curitiba, Brazil.

We thank Dr. Lisa Koenig, a board-certified oral and maxillofacial radiologist who interpreted the CBCT scans.

\section{References}

1. Cookson, A.M. Movement of an upper central incisor across the midline. $\mathrm{Br} \mathrm{J}$ Orthod. 1981; 8: 59-60

2. Follin, M. Orthodontic movement of maxillary incisor into the midline. Swed Dent J. 1985; 9: 913

3. Melnik, A.K. Orthodontic movement of a supplemental maxillary incisor through the midpalatal suture area. Am J Orthod Dentofacial Orthop. 1993; 104: 85-90

4. McCollum, A.G. Crossing the midline: a long-term case report. Am J Orthod Dentofacial Orthop. 1999;115: 559-562

5. Teufelberger, W., Schachner, P., Schicher-Kucher, N., and Bantleon, H.P. Long-term results of shifting a central incisor across the midline-case report. Inf Orthod Kieferorthop. 2008; 40: 239-247

6. (Persson M. Orthodontic movement of maxillary incisors through the midpalatal suture area: case report. Nordsk Orthodontiste Sällskap, Congress 1976, Unpublished results. Cited in: Follin M, Ericson I, Thilander B. Orthodontic movement of maxillary incisors through the midpalatal area: an experimental study in dogs)Eur J Orthod. 1984; 6: 237-246 
7. Follin, M., Ericsson, I., and Thilander, B. Orthodontic movement of maxillary incisors through the midpalatal suture area: an experimental study in dogs. Eur J Orthod. 1984; 6: 237-246

8. Tausche, E. and Harzer, W. Treatment of a patient with class II malocclusion, impacted maxillary canine with a dilacerated root, and peg-shaped lateral incisor. Am J Orthod Dentofacial Orthop. 2008;133: 762-770

9. Bosio, J.A. The incidence of maxillary sinus retention cyst in orthodontic patients. World J Orthod. 2009; 10: e7-e8

10. Mendes, R.A. and Rocha, G. Mandibular third molar autotransplantation - literature review with clinical cases. J Can Dent Assoc. 2004; 70: 761-766

11. Bishara, S.E. Clinical management of impacted maxillary canines. Semin Orthod. 1998; 4: 8798

12. Rosa, M. and Zachrisson, B.U. Integrating space closure and esthetic dentistry in patients with missing maxillary lateral incisors. J Clin Orthod. 2007; 49: 563-573

13. Spear, F.M., Kokich, V.G., and Mathews, D.P. Interdisciplinary management of anterior dental esthetics. J Am Dent Assoc. 2006; 137: 160-169

14. Spear, F.M. and Kokich, V.G. A multidisciplinary approach to esthetic dentistry. Dent Clin N Am. 2007; 51: 487-505

15. in: H. Sicher, E. Dubrul (Eds.) Oral anatomy. 6th ed. Mosby, St. Louis; 1975: 392

16. Rupprecht, R.D., Horning, G.M., Nicoll, B.K., and Cohen, M.E. Prevalence of dehiscences and fenestrations in modern American skulls. J Periodontol. 2001; 72: 722-729

17. Cairo, F., Pagliaro, U., and Nieri, M. Treatment of gingival recession with coronally advanced flap procedures: a systematic review. J Clin Periodontol. 2008; 35: 136-162

18. Thilander, B., Odman, J., and Lekholm, U. Orthodontic aspects of the use of oral implants in adolescents: a 10 year follow-up study. Eur J Orthod. 2001; 23: 715-731

19. Kokich, V.G. and Crabill, K.E. Managing the patient with missing or malformed maxillary central incisors. Am J Orthod Dentofacial Orthop. 2006; 129: S55-S63

20. Kokich, V.O. Jr. and Kinzer, G.A. Managing congenitally missing lateral incisors. Part I: canine substitution. J Esthet Restor Dent. 2005; 17: 5-10

21. Reynders, R.M. Orthodontics and temporomandibular disorders: a review of the literature (1966-1988). Am J Orthod Dentofacial Orthop. 1990; 97: 463-471 